\title{
A non-equilibrium thermodynamics model of multicomponent mass and heat transport in pervaporation processes
}

\author{
Juan P. G. Villaluenga and Signe Kjelstrup
}

Keywords. Pervaporation processes, multicomponent mass transfer, membrane processes, separation of water/ethanol mixtures.

\begin{abstract}
The framework of non-equilibrium thermodynamics (NET) is used to derive heat and mass transport equations for pervaporation of a binary mixture in a membrane. In this study, the assumption of equilibrium of the sorbed phase in the membrane and the adjacent phases at the feed and permeate sides of the membrane is abandoned, defining the interface properties using local equilibrium. The transport equations have been used to model the pervaporation of a water-ethanol mixture, which is typically encountered in the dehydration of organics. The water and ethanol activities and temperature profiles are calculated taking mass and heat coupling effects and surfaces into account. The NET approach is deemed good because the temperature results provided by the model are comparable to experimental results available for water-alcohol systems.
\end{abstract}

\section{Introduction}

Pervaporation is a membrane separation process in which one or more components of a liquid mixture permeate selectively through a dense membrane while they partially evaporate. The main driving force on a component going through pervaporation is the chemical potential gradient, obtained by partial pressure reduction on the permeate side. The driving force of other components, as well as a temperature gradient, may also contribute. It is difficult to formulate a single explanation for the transport process in per- 
vaporation because of the complicated permeant-permeant and permeantmembrane interactions, besides the phase transition from liquid to vapor. The liquid-vapor transition might well be rate limiting for the overall process in cases where large enthalpies of evaporation are involved. Particular attention should therefore be given to the energy balance, and how to include this. In addition, the absorption and desorption processes at the membrane surfaces have to be considered in the modeling of the overall process.

There are several approaches that describe mass transport in pervaporation: the solution-diffusion model, the pore-flow model, and the nonequilibrium thermodynamics approach. The solution-diffusion model was first adopted by Binning et al. to describe the mass transport through dense membranes in pervaporation, and has become the accepted pattern for this process by most researchers [1]. According to this model, pervaporation transport consists of three consecutive processes: selective sorption of the components from the feed liquid to the membrane surface, diffusion of the components in the membrane, and desorption of the components to the vapor phase on the permeate side of the membrane. One of the main criticisms of this model is due to the fact that the phase change occurring in the evaporation process - because the feed is in the liquid state whereas the permeate is in the vapor state - is not fully addressed. In general, the sorption and diffusion processes depend on the concentration of the components in the membrane; consequently, the solubility and diffusivity are concentrationdependent variables. A number of equations for mass transport have been formulated using different empirical expressions of concentration dependence of sorption and diffusion coefficients [2].

Matsuura and co-workers [3-5] proposed a transport model applicable to pervaporation on the basis of a pore-flow mechanism. In this model, it is assumed that there are bundles of straight cylindrical pores, which are in an isothermal condition, penetrating across the membrane. The distinguishing feature of the pore-flow model is that it assumes a liquid-vapor phase boundary inside the membrane, and the mass transport is considered to be a combination of liquid transport and vapor transport in series. Accordingly, the mass transport consists of three consecutive steps: (i) liquid transport from the pore inlet to a liquid-vapor phase boundary, (ii) evaporation at the phase boundary, and (iii) vapor transport from the boundary to the pore outlet. The applicability of the transport equations based on the pore-flow theory has been tested to analyze the effects of downstream pressure, temperature, and composition on the pervaporation performance of a variety of binary mixtures by using different polymeric membranes. One of the 
advantages of the pore-flow model over the solution-diffusion model is its simplicity and predictability.

The non-equilibrium thermodynamics (NET) theory allows modeling the coupling effects that occur between the individual permeants and the membrane, as well as between permeants. Hwang [6] applied the principles of thermodynamics of NET to analyze different membrane processes, including pervaporation, in homogeneous systems. Hwang developed a general guideline to describe a given membrane process from a theoretical standpoint, which yields flux equations with appropriate driving forces easily related to experimentally observable quantities. The author states that the key is to express the chemical potential in terms of more convenient variables for every specific membrane process. The author's mathematical treatment considered an isothermal multicomponent permeation process across a membrane, assuming a one-dimensional steady state problem for the sake of simplicity.

It is common in current modeling of membrane transport, to assume equilibrium between any two adjacent phases, i.e., the adsorbed phase in the membrane is assumed to be in equilibrium with the bulk fluids at any side. Only recently, Kjelstrup et al. [7-9] abandoned the assumption of equilibrium across the interfaces and defined the interface properties using local equilibrium instead. Inzoli et al. [10] have concluded from a nonequilibrium molecular dynamics study on the adsorption of n-butane gas on a silicalite- 1 membrane that the surface is rate limiting to heat transfer and that transport in zeolites should include a thermal driving force, in addition to the chemical driving force. It is likely that this is also the case in pervaporation, where a phase transition takes place from absorbed liquid to vapor. In the present study, we use the framework of NET and the concept of the dividing surface to derive transport equations for pervaporation, including a thermal driving force. Such a driving force is commonly disregarded in spite of evidence of thermal polarization and enhanced effects of temperature. Recently, Kuhn et al. [9] studied the coupled mass and heat transport processes in the pervaporation of pure water in a zeolitetype membrane using the framework of NET. Kuhn et al. found that the surface effects lead to discontinuities in the activity and temperature at the membrane interfaces. This study also revealed coupling effects between the mass and heat transport processes: the heat flux induced an extra driving force for mass transport, reducing the activity over the membrane, and the mass transport across the interfaces was governed by the coupling with the heat flux. In the present study, transport equations for the coupled heat and mass transport are derived to model the pervaporation of a binary mixture 
in a dense membrane. For simplicity, the equations for a twocomponent system are described, but the generalization to a multicomponent system is straightforward.

\section{Thermodynamic model equations}

The description of the multicomponent mass and heat transport in a heterogeneous system is based on a NET approach derived by Bedeaux and Kjelstrup [11]. The central part of a pervaporation system is the membrane cell, which is basically composed of the membrane and two adjacent solutions (feed and permeate mixtures). All these subsystems are considered as homogeneous phases. The feed and permeate homogeneous phases next to the membrane are indicated by superscripts $l$ and $r$ (left and right), respectively, and the membrane phase is indicated by superscript $m$. It should be pointed out that there are two components $(A$ and $B)$ in the feed and permeate mixtures, whereas in the membrane one really has three components $(A, B$, plus the membrane $C$ ), so the natural frame of reference for transport in the membrane system is the membrane itself. According to Bedeaux and Kjelstrup's approach, an interface is a separate thermodynamic system between the two adjacent phases [7]. Then, two dividing surfaces in a membrane cell can be defined: the liquid feed-membrane interface and the membrane-vapor permeate interface. By this procedure, the system is divided into three distinct phases: (i) the feed-membrane surface where transport processes are driven by differences in temperature and chemical potentials, (ii) the membrane where transport processes are driven by gradients in temperature and chemical potentials, and (iii) the membrane-permeate surface where again the transport processes are driven by differences in temperature and chemical potentials. In order to simplify the mathematical treatment, the analysis is limited to stationary state calculations and to a one-dimensional transport problem, with the membrane as the only homogeneous phase with transport.

We are interested in expressions for the variation in the temperature and the chemical potentials close to and at the interfaces. In the derivation of the equations describing transport processes of heat and mass, the NET model for homogeneous systems should be combined with NET approach for surfaces. The equations for the transport of heat and mass in each subsystem can be derived from the entropy production rate as explained below.

Thermal and chemical equilibrium between the feed and the membrane for the two components $A$ and $B$ in the liquid phase are not assumed, across 
the feed-membrane interface, because the enthalpies of sorption may be relatively large, and the thermal conductivities may be low. Across the membrane, we have coupled transports of heat and mass ( $A$ and $B$ relative to the membrane $C$ ) until the membrane-permeate interface, where the feeds evaporate. We do not assume equilibrium on the permeate side, across the membrane-vapor interface, since large enthalpies of desorption and evaporation are involved, and the thermal conductivity of the vapor is low. The terminology follows Kjelstrup and Bedeaux [11]. A single superscript is used to indicate the relevant subsystem. When a double superscript is used, the first gives the subsystem, and the second gives the nearest subsystem. For instance, $l, m$ means the feed solution close to the membrane.

\subsection{Feed-membrane surface}

For the feed-membrane interface, the excess entropy production, $\sigma^{l}$, in the left side (or feed-side) of the membrane surface can be written as [11]

$$
\begin{aligned}
\sigma^{l}=J_{q}^{\prime l} \Delta_{l, m} & \left(\frac{1}{T}\right) \\
& +J_{A}\left[-\frac{\Delta_{l, m} \mu_{A, T}\left(T^{m, l}\right)}{T^{m, l}}\right]+J_{B}\left[-\frac{\Delta_{l, m} \mu_{B, T}\left(T^{m, l}\right)}{T^{m, l}}\right],
\end{aligned}
$$

where $J_{q}^{\prime l}$ is the measurable heat flux in the feed side, and $J_{i}$ is the molar flux of component $i(i=A, B)$. This notation is based on the two phases adjacent to the surface. Each jump is written as the value to the right minus the value to the left. This choice gives the jumps the same sign as the gradients in the homogeneous phases for increasing or decreasing variables. The subscripts of $\Delta$ refer to the two locations between which the difference is taken.

According to Kjelstrup and Bedeaux's approach, expressions for the driving forces as function of the fluxes can be derived from the entropy production:

$$
\begin{array}{r}
\Delta_{l, m}\left(\frac{1}{T}\right)=r_{q q}^{s, l} J_{q}^{\prime l}+r_{q A}^{s, l} J_{A}+r_{q B}^{s, l} J_{B}, \\
-\frac{\Delta_{l, m} \mu_{A, T}\left(T^{m, l}\right)}{T^{m, l}}=r_{A q}^{s, l} J_{q}^{\prime l}+r_{A A}^{s, l} J_{A}+r_{A B}^{s, l} J_{B}, \\
-\frac{\Delta_{l, m} \mu_{B, T}\left(T^{m, l}\right)}{T^{m, l}}=r_{B q}^{s, l} J_{q}^{\prime l}+r_{B A}^{s, l} J_{A}+r_{B B}^{s, l} J_{B} .
\end{array}
$$


The transport equations derived from the equations given above are

$$
\begin{aligned}
\Delta_{l, m} T & =-\frac{1}{\lambda^{s, l}}\left(J_{q}^{\prime l}-J_{A} q_{A}^{* s, l}-J_{B} q_{B}^{* s, l}\right), \\
\frac{\Delta_{l, m} \mu_{A, T}\left(T^{m, l}\right)}{T^{m, l}} & =-\frac{q_{A}^{* s, l}}{T^{l, m} T^{m, l}} \Delta_{l, m} T-R_{A A}^{s, l} J_{A}-R_{A B}^{s, l} J_{B}, \\
\frac{\Delta_{l, m} \mu_{B, T}\left(T^{m, l}\right)}{T^{m, l}} & =-\frac{q_{B}^{* s, l}}{T^{l, m} T^{m, l}} \Delta_{l, m} T-R_{B A}^{s, l} J_{A}-R_{B B}^{s, l} J_{B} .
\end{aligned}
$$

There are seven transport coefficients, which have to be either experimentally determined or theoretically estimated. The thermal conductivity is defined as

$$
\lambda^{s, l}=-\left[\frac{J_{q}^{\prime l}}{\Delta_{l, m} T}\right]_{J_{A}=0, J_{B}=0}=\frac{1}{\left(T^{l, m}\right)^{2} r_{q q}^{s, l}} .
$$

The heat of transfer in the surface is defined as

$$
q_{i}^{* s, l}=\left[\frac{J_{q}^{\prime l}}{J_{i}}\right]_{\Delta_{l, m} T=0, J_{j}=0}=-\frac{r_{q i}^{s, l}}{r_{q q}^{s, l}} \quad(i, j=A, B),(i \neq j) .
$$

The four resistivity coefficients are defined as

$$
R_{i j}^{s, l}=r_{i j}^{s, l}-\frac{r_{i q}^{s, l} r_{q j}^{s, l}}{r_{q q}^{s, l}} \quad(i, j=A, B) .
$$

\subsection{Membrane}

The entropy production for the coupled transport of heat and mass can be obtained as the sum of the product of the thermodynamic driving forces and conjugate fluxes. There are then three independent driving forces in the membrane phase, one thermal and two component driving forces. The entropy production results in

$$
\sigma^{m}=J_{q}^{\prime m} \frac{\mathrm{d}}{\mathrm{d} x}\left(\frac{1}{T}\right)-J_{A} \frac{1}{T} \frac{\mathrm{d} \mu_{A, T}}{\mathrm{~d} x}-J_{B} \frac{1}{T} \frac{\mathrm{d} \mu_{B, T}}{\mathrm{~d} x},
$$

where $J_{q}^{\prime m}$ is the measurable heat flux in the membrane. The chemical potential should be differentiated at constant temperature, giving $\mathrm{d} \mu_{i, T}$. The molar fluxes, but not the observable heat flux, are constant throughout the membrane. The force-flux equations can be derived from the entropy 
production. Choosing as variables some coefficients that are practical to measure, the differential form for the heat transport equation can be written as follows:

$$
\frac{d T}{\mathrm{~d} x}=-\frac{1}{\lambda^{m}}\left(J_{q}^{\prime m}-q_{A}^{* m} J_{A}-q_{B}^{* m} J_{B}\right) .
$$

The mass transport equations can be expressed as

$$
\begin{aligned}
& \frac{c_{A}}{T} \frac{\mathrm{d} \mu_{A, T}}{\mathrm{~d} x}=-\frac{c_{A} q_{A}^{* m}}{T^{2}} \frac{\mathrm{d} T}{\mathrm{~d} x}-\frac{R_{A A}}{c_{A}} J_{A}-\frac{R_{A B}}{c_{B}} J_{B}, \\
& \frac{c_{B}}{T} \frac{\mathrm{d} \mu_{B, T}}{\mathrm{~d} x}=-\frac{c_{B} q_{B}^{* m}}{T^{2}} \frac{\mathrm{d} T}{\mathrm{~d} x}-\frac{R_{B A}}{c_{A}} J_{A}-\frac{R_{B B}}{c_{B}} J_{B} .
\end{aligned}
$$

The thermal conductivity at zero mass flow, $\lambda^{m}$, and the measurable heat of transfer in the membrane, $q_{i}^{* m}$, at constant temperature are defined as

$$
\begin{aligned}
\lambda^{m} & \equiv-\left[\frac{J_{q}^{\prime m}}{\mathrm{~d} T / \mathrm{d} x}\right]_{J_{A}=J_{B}=0}, \\
q_{i}^{* m} & \equiv\left[\frac{J_{q}^{\prime m}}{J_{i}}\right]_{d T=0, J_{j}=0} \quad(i, j=A, B),(i \neq j) .
\end{aligned}
$$

The resistivity coefficients $R_{i j}$ for the component fluxes can be related to the phenomenological coefficients $L_{i j}$, as Kjelstrup and Bedeaux showed [11]:

$$
\begin{aligned}
R_{A A} & =\frac{L_{B B}}{L_{A A} L_{B B}-L_{B A} L_{A B}}, \\
R_{B B} & =\frac{L_{A A}}{L_{A A} L_{B B}-L_{B A} L_{A B}}, \\
R_{A B}=R_{B A} & =\frac{-L_{A B}}{L_{A A} L_{B B}-L_{B A} L_{A B}} .
\end{aligned}
$$

The phenomenological coefficients are related to the diffusion coefficients $D_{i j}$ in the membrane frame of reference as follows [11]:

$$
\begin{aligned}
& D_{A A}=L_{A A} c_{A} R, \\
& D_{B B}=L_{B B} c_{B} R, \\
& D_{A B}=L_{A B} c_{A} R, \\
& D_{B A}=L_{B A} c_{B} R .
\end{aligned}
$$


For convenience, the activity can also be introduced as variable using

$$
\frac{\mathrm{d} \mu_{i, T}}{\mathrm{~d} x}=\frac{R T}{a_{i}} \frac{\mathrm{d} a_{i}}{\mathrm{~d} x}
$$

Then, the mass transport equation can be expressed as follows:

$$
\begin{aligned}
\frac{\mathrm{d} a_{A}}{\mathrm{~d} x} & =-\frac{a_{A} q_{A}^{* m}}{R T^{2}} \frac{\mathrm{d} T}{\mathrm{~d} x}-\frac{a_{A} R_{A A}}{R c_{A}^{2}} J_{A}-\frac{a_{A} R_{A B}}{R c_{A} c_{B}} J_{B}, \\
\frac{\mathrm{d} a_{B}}{\mathrm{~d} x} & =-\frac{a_{B} q_{B}^{* m}}{R T^{2}} \frac{\mathrm{d} T}{\mathrm{~d} x}-\frac{a_{B} R_{B A}}{R c_{A} c_{B}} J_{A}-\frac{a_{B} R_{B B}}{R c_{B}^{2}} J_{B} .
\end{aligned}
$$

Equations (7), (15), and (16) have to be solved numerically with knowledge of the transport coefficients together with the appropriate boundary conditions.

\subsection{Membrane-permeate surface}

The entropy production, $\sigma^{r}$, in the right-side (or permeate-side) membrane surface is

$$
\begin{aligned}
\sigma^{r}=J_{q}^{\prime r} \Delta_{m, r} & \left(\frac{1}{T}\right) \\
& +J_{A}\left[-\frac{\Delta_{m, r} \mu_{A, T}\left(T^{m, r}\right)}{T^{m, r}}\right]+J_{B}\left[-\frac{\Delta_{m, r} \mu_{B, T}\left(T^{m, r}\right)}{T^{m, r}}\right],
\end{aligned}
$$

where $J_{q}^{\prime r}$ is the measurable heat flux in the permeate side. The linear relations between the forces and the fluxes are as follows:

$$
\begin{array}{r}
\Delta_{m, r}\left(\frac{1}{T}\right)=r_{q q}^{s, r} J_{q}^{\prime r}+r_{q A}^{s, r} J_{A}+r_{q B}^{s, r} J_{B}, \\
-\frac{\Delta_{m, r} \mu_{A, T}\left(T^{m, r}\right)}{T^{m, r}}=r_{A q}^{s, r} J_{q}^{\prime r}+r_{A A}^{s, r} J_{A}+r_{A B}^{s, r} J_{B}, \\
-\frac{\Delta_{m, r} \mu_{B, T}\left(T^{m, r}\right)}{T^{m, r}}=r_{B q}^{s, r} J_{q}^{\prime r}+r_{B A}^{s, r} J_{A}+r_{B B}^{s, r} J_{B} .
\end{array}
$$

The transport equations derived from the equations given above are

$$
\begin{gathered}
\Delta_{m, r} T=-\frac{1}{\lambda^{s, r}}\left(J_{q}^{\prime r}-J_{A} q_{A}^{* s, r}-J_{B} q_{B}^{* s, r}\right), \\
\frac{\Delta_{m, r} \mu_{A, T}\left(T^{m, r}\right)}{T^{m, r}}=-\frac{q_{A}^{* s, r}}{T^{r, m} T^{m, r}} \Delta_{m, r} T-R_{A A}^{s, r} J_{A}-R_{A B}^{s, r} J_{B}, \\
\frac{\Delta_{m, r} \mu_{B, T}\left(T^{m, r}\right)}{T^{m, r}}=-\frac{q_{B}^{* s, r}}{T^{r, m} T^{m, r}} \Delta_{m, r} T-R_{B A}^{s, r} J_{A}-R_{B B}^{s, r} J_{B} .
\end{gathered}
$$


The transport coefficients have to be either experimentally determined or theoretically estimated. The thermal conductivity is defined as

$$
\lambda^{s, r}=-\left[\frac{J_{q}^{\prime r}}{\Delta_{m, r} T}\right]_{J_{A}=0, J_{B}=0}=\frac{1}{\left(T^{m, r}\right)^{2} r_{q q}^{s, r}} .
$$

The heat of transfer in the surface is defined as

$$
q_{i}^{* s, r}=\left[\frac{J_{q}^{\prime r}}{J_{i}}\right]_{\Delta_{m, r} T=0, J_{j}=0}=-\frac{r_{q i}^{s, r}}{r_{q q}^{s, r}} \quad(i, j=A, B),(i \neq j) .
$$

There are four resistivity coefficients defined as

$$
R_{i j}^{s, r}=r_{i j}^{s, r}-\frac{r_{i q}^{s, r} r_{q j}^{s, r}}{r_{q q}^{s, r}} \quad(i, j=A, B)
$$

\section{A numerical example}

In the present study dehydration of ethanol is analyzed [12]. The system is assumed to be in a stationary state. In this case, the total heat flux, $J_{q}$, the water (component $A$ ), and ethanol (component $B$ ) fluxes are constant throughout the system. Then, the following expression applies:

$$
\begin{aligned}
J_{q} & =J_{q}^{\prime l}+H_{A}^{l} J_{A}+H_{B}^{l} J_{B} \\
& =J_{q}^{\prime m}+H_{A}^{m} J_{A}+H_{B}^{m} J_{B}=J_{q}^{\prime r}+H_{A}^{r} J_{A}+H_{B}^{r} J_{B} .
\end{aligned}
$$

In addition, the following expressions between the measurable heat fluxes in each subsystem can be obtained:

$$
\begin{aligned}
& J_{q}^{\prime l}=J_{q}^{\prime m}+J_{A} \Delta_{l, m} H_{A}+J_{B} \Delta_{l, m} H_{B}, \\
& J_{q}^{\prime m}=J_{q}^{\prime r}+J_{A} \Delta_{m, r} H_{A}+J_{B} \Delta_{m, r} H_{B} .
\end{aligned}
$$

The measurable heat fluxes only can change at the interfaces, where the water and ethanol undergoes an enthalpy change. The enthalpy change at the feed liquid-membrane interface is denoted $\Delta_{l, m} H_{i}$, whereas the enthalpy change at the membrane-permeate interface is denoted $\Delta_{m, r} H_{i}$. It is worth noting that two thermal processes have to be considered at the membrane surfaces in a pervaporation process. Firstly, sorption of components at the feed-membrane surface, and desorption of components at the membranepermeate surface are both associated with an enthalpy change. Secondly, there is a phase change occurring in the system, because the components 
are in the liquid state in the feed side and in the gas state in the permeate side. Therefore, the enthalpies of sorption and desorption, and the enthalpy of the phase transition have to be included in the estimation of the heat effects in the system.

\subsection{Feed-membrane interface}

Let us consider a binary mixture of water-ethanol with a water content of $10 \mathrm{wt} . \%$. The activity coefficients of water, $a_{A}^{l, m}\left(T^{l, m}\right)$, and ethanol, $a_{B}^{l, m}\left(T^{l, m}\right)$, in the feed mixture are 0.2213 and 0.8093 , respectively. The values of water and ethanol molar fluxes are given in Table 1 (see [12-14]). The temperature, $T^{l, m}$, of the feed mixture is set to $308 \mathrm{~K}$. The value of the thermal conductivity in the $l$-side membrane surface, $\lambda^{s, l}$, which is estimated with Eq. (4) based on reported values of the coefficient $r_{q q}^{s, l}$ (see $[9,15])$, is given in Table 1 . The enthalpy change, $\Delta_{l, m} H_{i}$, values of water and ethanol are set to, respectively, $-3.0 \mathrm{~kJ} \cdot \mathrm{mol}^{-1}$ and $2.1 \mathrm{~kJ} \cdot \mathrm{mol}^{-1}$, which are their sorption enthalpies at the feed-membrane surface [16-19]. The heat of transfer of water, $q_{A}^{* s, l}$, and ethanol, $q_{B}^{* s, l}$, were estimated as a fraction of the enthalpy change in the interface as follows $[9,11]$ :

$$
q_{i}^{* s, l}=-k \Delta_{l, m} H_{i},
$$

where $k$ is a scaling coefficient. Dimensionless scaling parameters are introduced to compare the interfacial transfer coefficients with the corresponding coefficients in the adjacent homogeneous system. The scaling coefficients show how effective the surface is as a barrier for transport. Previous studies on water pervaporation $[9,20]$ gave $k$ equal to 0.3 . The values of heat of transfer of water and ethanol in the $l$-surface, using $k=0.3$, are given in Table 1 . Because of the novelty of the concept of the dividing surface, the required resistivity parameters $R_{i j}^{s, l}$ are not available for the system analyzed in the present study. The transport parameters are estimated based on simulation data and theory, and the values correspond to values found for other systems, because actual data are not available. Hence, we have considered the following procedure to estimate the resistivity coefficients in the surfaces. The values of $r_{q q}^{s, l}$ and $r_{i j}^{s, l}$ were determined experimentally $[21,22]$ or estimated by molecular simulations $[10,15,23]$. The value of $q_{i}^{* s, l}$ was estimated as a fraction of the enthalpy change in the interface. Then, the values of resistivity coefficients $r_{i q}^{s, l}$ are estimated with Eq. (5). Finally, the values of the resistivity coefficients $R_{i j}^{s, l}$ in the feed-membrane interface were estimated with Eq. (6). The estimated values of $R_{i j}^{s, l}$ are given in Table 1. 


\begin{tabular}{lll}
\hline Parameter & Units & Value \\
\hline$J_{A}$ & $\mathrm{~mol} \cdot \mathrm{m}^{-2} \cdot \mathrm{s}^{-1}$ & 0.035 \\
$J_{B}$ & $\mathrm{~mol} \cdot \mathrm{m}^{-2} \cdot \mathrm{s}^{-1}$ & $5.0 \times 10^{-4}$ \\
$\lambda^{s, l}$ & $\mathrm{~W} \cdot \mathrm{m}^{-2} \cdot \mathrm{K}^{-1}$ & $10^{3}$ \\
$J_{q}^{\prime l}$ & $\mathrm{~W} \cdot \mathrm{m}^{-2}$ & 3000 \\
$q_{A}^{* s, l}$ & $\mathrm{~J} \cdot \mathrm{mol}^{-1}$ & 900 \\
$q_{B}^{* s, l}$ & $\mathrm{~J} \cdot \mathrm{mol}^{-1}$ & -630 \\
$R_{A A}^{s, l}$ & $\mathrm{~J} \cdot \mathrm{m}^{2} \cdot \mathrm{s} \cdot \mathrm{mol}^{-2} \cdot \mathrm{K}^{-1}$ & $1 \times 10^{-5}$ \\
$R_{B B}^{s, l}$ & $\mathrm{~J} \cdot \mathrm{m}^{2} \cdot \mathrm{s} \cdot \mathrm{mol}^{-2} \cdot \mathrm{K}^{-1}$ & $3 \times 10^{-4}$ \\
$R_{A B}^{s, l}$ & $\mathrm{~J} \cdot \mathrm{m}^{2} \cdot \mathrm{s} \cdot \mathrm{mol}^{-2} \cdot \mathrm{K}^{-1}$ & $8 \times 10^{-4}$ \\
\hline
\end{tabular}

Table 1. Transport parameters for the feed-membrane interface, $l$-side of the membrane.

The value of the measurable heat flux in the membrane feed side is estimated based on reported data of the temperature difference between the bulk of the feed mixture and the membrane surface, at the permeate side, for pervaporation dehydration of propanol $[24,25]$. In the present study, the measurable heat flux in the $l$-side is set to $3 \times 10^{3} \mathrm{~W} \cdot \mathrm{m}^{-2}$.

Once the transport coefficients are calculated, the change in the temperature and the chemical potentials of components $A$ and $B$ were estimated using Eqs. (1), (2), and (3). The values are $-3.0 \mathrm{~K}, 8.85 \mathrm{~J} / \mathrm{mol}$, and $-6.20 \mathrm{~J} / \mathrm{mol}$, respectively. A substantial sizable effect for the temperature at the feed-membrane surface is observed, as shown in Figure 1. The temperature, $T^{m, l}$, is $305 \mathrm{~K}$. The chemical potential changes can be converted into activity variation by using the following relations:

$$
\begin{aligned}
& \Delta_{l, m} \mu_{A, T}\left(T^{m, l}\right)=R T^{m, l} \ln \left[\frac{a_{A}^{m, l}\left(T^{m, l}\right)}{a_{A}^{l, m}\left(T^{m, l}\right)}\right], \\
& \Delta_{l, m} \mu_{B, T}\left(T^{m, l}\right)=R T^{m, l} \ln \left[\frac{a_{B}^{m, l}\left(T^{m, l}\right)}{a_{B}^{l, m}\left(T^{m, l}\right)}\right] .
\end{aligned}
$$

The activities $a_{A}^{l, m}\left(T^{m, l}\right)$ and $a_{B}^{l, m}\left(T^{m, l}\right)$ defined at the local temperature $T^{m, l}$ can be obtained from the activities $a_{A}^{l, m}\left(T^{l, m}\right)$ and $a_{B}^{l, m}\left(T^{m, l}\right)$ at the 


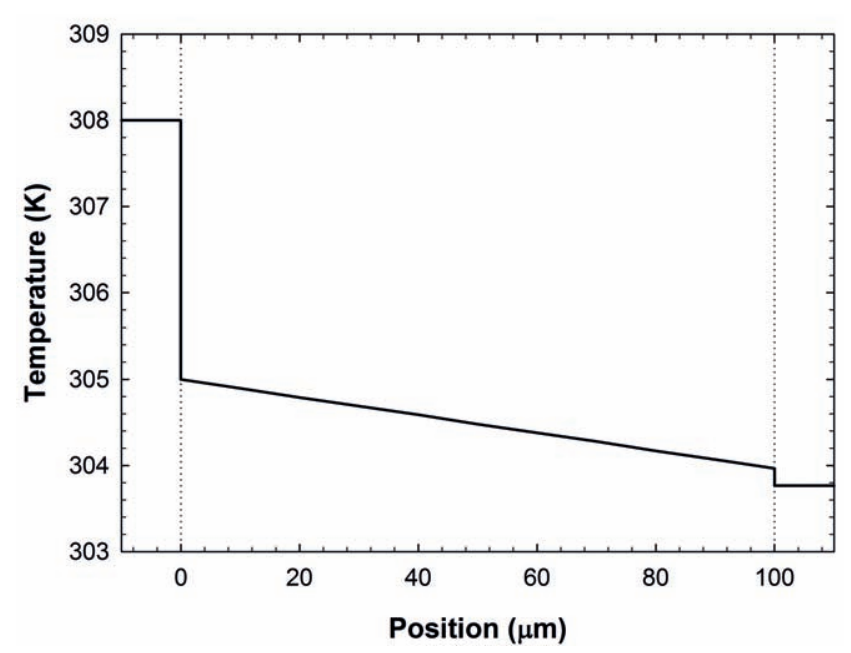

Figure 1. Temperature profile across the feed liquid solution, the membrane, and the permeate vapor mixture. The vertical dashed lines indicate, from left to right, the liquid-membrane interface and the membrane-permeate interface.

$l$-side of the surface defined at $T^{l, m}$ using the Gibbs-Helmholtz relation:

$$
\begin{aligned}
& R \ln \left[\frac{a_{A}^{l, m}\left(T^{m, l}\right)}{a_{A}^{l, m}\left(T^{l, m}\right)}\right]=\Delta_{l, m} H_{A} \frac{\Delta_{l, m} T}{T^{l, m} T^{m, l}}, \\
& R \ln \left[\frac{a_{B}^{l, m}\left(T^{m, l}\right)}{a_{B}^{l, m}\left(T^{l, m}\right)}\right]=\Delta_{l, m} H_{B} \frac{\Delta_{l, m} T}{T^{l, m} T^{m, l}} .
\end{aligned}
$$

The calculations using Eqs. (27) and (28) give 0.2239 and 0.8028 as the activities $a_{A}^{l, m}\left(T^{m, l}\right)$ and $a_{B}^{l, m}\left(T^{m, l}\right)$, respectively. These values are used in Eqs. (25) and (26) to calculate the activity of water, $a_{A}^{m, l}\left(T^{m, l}\right)$, and ethanol, $a_{B}^{m, l}\left(T^{m, l}\right)$, in the membrane close to the feed surface. The values so obtained are, respectively, 0.2247 and 0.8008 . It then follows that, at the feed-membrane surface, there is a small positive jump in the water activity, while the ethanol activity shows a little drop.

\subsection{Membrane}

The value for the thermal conductivity, $\lambda^{m}$, of the membrane is set to $0.3 \mathrm{~W} \cdot \mathrm{m}^{-1} \cdot \mathrm{K}^{-1}$ (see [26-28]). The heat of transfer of water and ethanol in the membrane were estimated from reported data on thermo-osmosis of 


\begin{tabular}{lll}
\hline Parameter & Units & Value \\
\hline$\delta$ & $\mathrm{m}$ & $1.0 \times 10^{-4}$ \\
$\lambda^{m}$ & $\mathrm{~W} \cdot \mathrm{m}^{-1} \cdot \mathrm{K}^{-1}$ & 0.3 \\
$J_{q}^{\prime m}$ & $\mathrm{~W} \cdot \mathrm{m}^{-2}$ & 3104 \\
$q_{A}^{* m}$ & $\mathrm{~J} \cdot \mathrm{mol}^{-1}$ & 14.6 \\
$q_{B}^{* m}$ & $\mathrm{~J} \cdot \mathrm{mol}^{-1}$ & 18.1 \\
$R_{A A}$ & $\mathrm{~J} \cdot \mathrm{s} \cdot \mathrm{m}^{-5} \cdot \mathrm{K}^{-1}$ & $2.0 \times 10^{13}$ \\
$R_{B B}$ & $\mathrm{~J} \cdot \mathrm{s} \cdot \mathrm{m}^{-5} \cdot \mathrm{K}^{-1}$ & $3.0 \times 10^{16}$ \\
$R_{A B}$ & $\mathrm{~J} \cdot \mathrm{s} \cdot \mathrm{m}^{-5} \cdot \mathrm{K}^{-1}$ & $-2.1 \times 10^{13}$ \\
\hline
\end{tabular}

Table 2. Transport parameters for the membrane.

mixtures of water and methanol in polymeric membranes [29]. The value of the measurable heat flux in the membrane which was calculated by using Eq. (23) is given in Table 2. The observable heat flux in the membrane is nearly the same as the observable heat flux in the feed. The phenomenological coefficients $L_{i j}(i, j=A, B)$ were calculated by using Eqs. (11), (12), (13), and (14) based on reported values of diffusion coefficients $D_{i j}$ $(i, j=A, B)$ and component concentration $c_{i}(i=A, B)$ in the membrane [2,13,14,19,30-32]. Then, the resistivity coefficients $R_{A A}, R_{B B}$, and $R_{A B}$ were estimated by using Eqs. (8), (9), and (10). Table 2 gives the values of the resistivity coefficients used in the calculations.

Once the transport coefficient values were known, Eqs. (7), (15), and (16) were solved simultaneously (by a numerical finite element method using MATLAB language) subject to the following boundary conditions at $x=0$ :

$$
\begin{aligned}
T & =T^{m, l}, \\
a_{A} & =a_{A}^{m, l}\left(T^{m, l}\right), \\
a_{B} & =a_{B}^{m, l}\left(T^{m, l}\right) .
\end{aligned}
$$

Figures 1, 2, and 3 show temperature and activity profiles in the complete membrane system, including liquid feed solution and permeate vapor mixture. The temperature varied linearly across the membrane as can be seen in Figure 1. A linear variation across the membrane in the activity of water and ethanol is also found, as shown in Figures 2 and 3, respectively. 


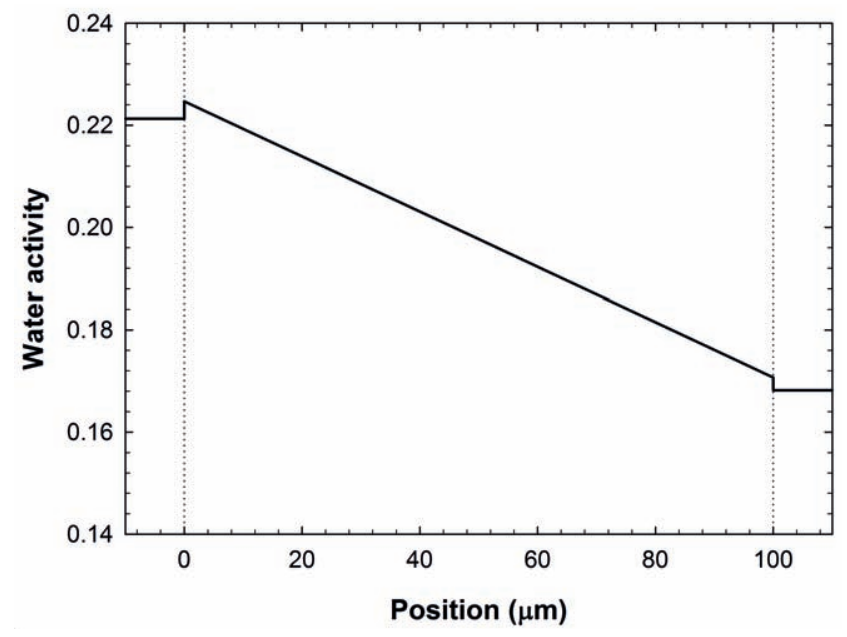

Figure 2. Water activity profile across the feed liquid solution, the membrane, and the permeate vapor mixture. The vertical dashed lines indicate, from left to right, the liquid-membrane interface and the membrane-permeate interface.

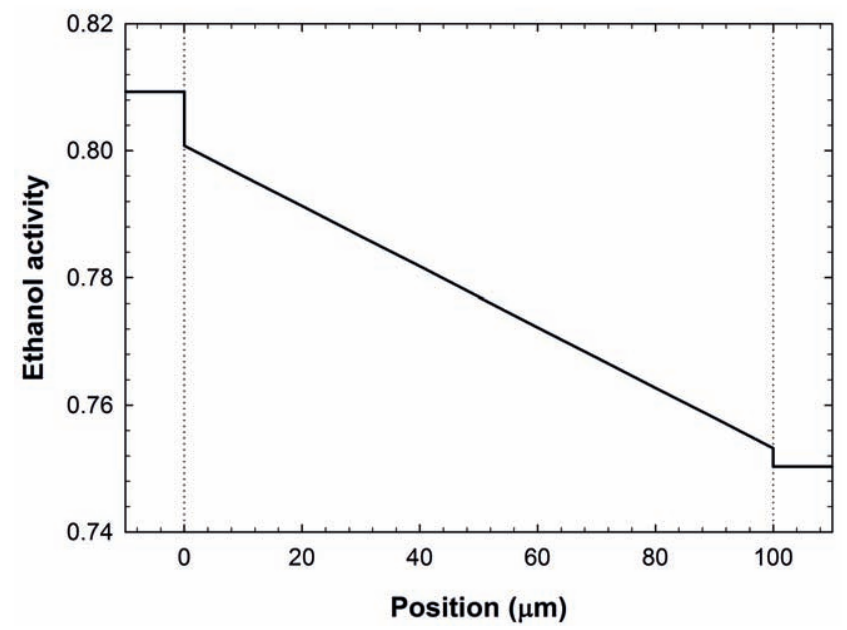

Figure 3. Ethanol activity profile across the feed liquid solution, the membrane, and the permeate vapor mixture. The vertical dashed lines indicate, from left to right, the liquid-membrane interface and the membranepermeate interface. 


\subsection{Membrane-permeate interface}

Because of the novelty of the concept of the dividing surface, we have a rather limited knowledge of the surface thermal conductivity $\lambda^{s, r}$. This transport parameter is estimated based on reported values from non-equilibrium molecular dynamic simulations of a vapor-liquid interface [15]. The heat of transfer of water and ethanol were estimated as a fraction of the enthalpy change in the surface,

$$
q_{i}^{* s, r}=-k \Delta_{m, r} H_{i} .
$$

The enthalpies of desorption and sorption are assumed to be numerically equal, but with different signs. The enthalpies of vaporization for the transition liquid to vapor for water and ethanol are set to $40.6 \mathrm{~kJ} \cdot \mathrm{mol}^{-1}$ and $38.6 \mathrm{~kJ} \cdot \mathrm{mol}^{-1}$, respectively. If we assume that the phase transition of the components occurs at the membrane permeate surface, it follows that the total enthalpy change $\left(\Delta_{m, r} H_{i}\right)$ values of water and ethanol at the membrane $r$-side becomes $43.6 \mathrm{~kJ} \cdot \mathrm{mol}^{-1}$ and $36.5 \mathrm{~kJ} \cdot \mathrm{mol}^{-1}$, respectively. The values of $q_{A}^{* s, r}$ and $q_{B}^{* s, r}$, using $k=0.3$, are given in Table 3 . The resistivity coefficients in the membrane-permeate interface $R_{i j}^{s, r}$ were estimated in a similar manner as followed in the estimation of the resistivity coefficient in the feed-membrane interface. The values were estimated using Eqs. (20), (21), and (22) based on values of $r_{i j}^{s, r}, r_{i q}^{s, r}$ and $r_{q q}^{s, r}$ reported in previous papers [9-11,15,21-23]. The values of the resistivities $R_{i j}^{s, r}$ used in the calculations are given in Table 3. The measurable heat flux in the surface was estimated from the values of observable heat flux in the membrane by using Eq. (24). As can be seen in Table 3, the observable heat flux in the membrane is significantly larger than that in the permeate side. At the membrane-permeate interface, the permeating components vaporize and desorb into the gas phase, requiring an enthalpy.

The values of $T^{m, r}, a_{A}^{m, r}\left(T^{m, r}\right)$, and $a_{B}^{m, r}\left(T^{m, r}\right)$ in the membrane close to the permeate surface were estimated from the functions $T(x), a_{A}(x)$, and $a_{B}(x)$ obtained in the previous section, by setting $x=\delta$ (membrane thickness). Their values are, respectively, $303.97 \mathrm{~K}, 0.1707$, and 0.7532 . The temperature jump $\Delta_{m, r} T$ can be calculated by using Eq. (17). If $J_{q}^{\prime r}=1560 \mathrm{~W} \cdot \mathrm{m}^{-2}, \Delta_{m, r} T$ becomes $-0.02 \mathrm{~K}$. Then, $T^{r, m}=T^{m, r}+$ $\Delta_{m, r} T=303.77 \mathrm{~K}$. A small sizable effect for the temperature is observed at the membrane-permeate surface. Figure 1 shows a temperature difference over the whole membrane system of $4.2 \mathrm{~K}$. To illustrate the impact of the surface, it is noted that the largest temperature drop is observed at the feed-membrane interface, because $71 \%$ of the temperature drop over 


\begin{tabular}{lll}
\hline Parameter & Units & Value \\
\hline$\lambda^{s, r}$ & $\mathrm{~W} \cdot \mathrm{m}^{-2} \cdot \mathrm{K}^{-1}$ & $10^{4}$ \\
$J_{q}^{\prime r}$ & $\mathrm{~W} \cdot \mathrm{m}^{-2}$ & 1560 \\
$q_{A}^{* s, r}$ & $\mathrm{~J} \cdot \mathrm{mol}^{-1}$ & $-13.1 \times 10^{3}$ \\
$q_{B}^{* s, r}$ & $\mathrm{~J} \cdot \mathrm{mol}^{-1}$ & $-10.9 \times 10^{3}$ \\
$R_{A A}^{s, r}$ & $\mathrm{~J} \cdot \mathrm{m}^{2} \cdot \mathrm{s} \cdot \mathrm{mol}^{-2} \cdot \mathrm{K}^{-1}$ & $1 \times 10^{-6}$ \\
$R_{B B}^{s, r}$ & $\mathrm{~J} \cdot \mathrm{m}^{2} \cdot \mathrm{s} \cdot \mathrm{mol}^{-2} \cdot \mathrm{K}^{-1}$ & $3 \times 10^{-5}$ \\
$R_{A B}^{s, r}$ & $\mathrm{~J} \cdot \mathrm{m}^{2} \cdot \mathrm{s} \cdot \mathrm{mol}^{-2} \cdot \mathrm{K}^{-1}$ & $8 \times 10^{-5}$ \\
\hline
\end{tabular}

Table 3. Transport parameters for the membrane-permeate interface, $r$-side of the membrane.

the system is generated at this surface. The temperature difference over the membrane is the $24 \%$ of the total difference. Whereas the temperature drop at the membrane-permeate interface is only the $5 \%$ of the total difference. These facts indicate that temperature polarization occurring in a pervaporation process may be caused to a larger extent by surface effects.

The jump in the chemical potentials

$$
\Delta_{m, r} \mu_{A, T}\left(T^{m, r}\right) \quad \text { and } \quad \Delta_{m, r} \mu_{B, T}\left(T^{m, r}\right),
$$

which were estimated by using Eqs. (18) and (19), is $-8.73 \mathrm{~J} \cdot \mathrm{mol}^{-1}$ and $-7.26 \mathrm{~J} \cdot \mathrm{mol}^{-1}$, respectively. The chemical potential change can be converted into activity variation by using the following relations:

$$
\begin{aligned}
& \Delta_{m, r} \mu_{A, T}\left(T^{m, r}\right)=R T^{m, r} \ln \left[\frac{a_{A}^{r, m}\left(T^{m, r}\right)}{a_{A}^{m, r}\left(T^{m, r}\right)}\right], \\
& \Delta_{m, r} \mu_{B, T}\left(T^{m, r}\right)=R T^{m, r} \ln \left[\frac{a_{B}^{r, m}\left(T^{m, r}\right)}{a_{B}^{m, r}\left(T^{m, r}\right)}\right] .
\end{aligned}
$$

It then follows that the activity of water, $a_{A}^{r, m}\left(T^{m, r}\right)$, and the activity of ethanol, $a_{B}^{r, m}\left(T^{m, r}\right)$, in the permeate close to the membrane surface are, respectively, 0.1701 and 0.7510 . We can correct the activities defined at the local temperature $T^{m, r}$ to the activities at the $r$-side of the surface defined at $T^{r, m}$, by

$$
R \ln \left[\frac{a_{A}^{r, m}\left(T^{r, m}\right)}{a_{A}^{r, m}\left(T^{m, r}\right)}\right]=\Delta_{m, r} H_{A} \frac{\Delta_{m, r} T}{T^{m, r} T^{r, m}},
$$




$$
R \ln \left[\frac{a_{B}^{r, m}\left(T^{r, m}\right)}{a_{B}^{r, m}\left(T^{m, r}\right)}\right]=\Delta_{m, r} H_{B} \frac{\Delta_{m, r} T}{T^{m, r} T^{r, m}} .
$$

Therefore, the activities $a_{A}^{r, m}\left(T^{r, m}\right)$ and $a_{B}^{r, m}\left(T^{r, m}\right)$, using Eqs. (32) and (33), are 0.1682 and 0.7503 , respectively. In contrast to temperature behavior, the largest activity difference over the whole system is found over the membrane itself. In the case of water, Figure 2 shows a positive jump of $6 \%$ in the activity of water at the feed-membrane interface; the activity shows a drop of $3 \%$ at the membrane-permeate interface, while there is a $91 \%$ decrease in activity over the membrane. In the case of ethanol, Figure 3 shows that the activity decreases by $14 \%$ of the total difference at the feed-membrane interface, and there is a decrease over the membrane, $81 \%$ of the total temperature difference, whereas the activity decreases by $5 \%$ of the total difference at the membrane-permeate interface. In both cases, Figures 2 and 3 show that the activity variations at the feed-membrane and membrane-permeate interfaces are not negligible, indicating that chemical equilibriums cannot be assumed at both sides of the membrane.

\section{Effect of membrane thickness}

To investigate the influence of the variation of the membrane thickness on mass and heat transport, the transport equations (7), (15), and (16) were solved in membranes with different thickness (in $\mu \mathrm{m}$ : 10, 25, 50, 100, and 150) using the same transport parameters, and the same boundary conditions at the membrane feed side (Eqs. (29), (30), and (31)). Table 4 gives the relative contributions to temperature difference over the membrane system. When the temperature difference over the membrane is compared to the temperature difference over the membrane surfaces, a significant contribution to the temperature drop is found at the feed-membrane surface. Moreover, the effect of the surface becomes larger as the membrane thickness decreases. As can be seen, $63 \%$ of the temperature drop over the system is generated at this surface of the thickest membrane, while $91 \%$ of the temperature drop is generated at this surface of the thinnest membrane. The temperature drop at the membrane-permeate surface is not significant. The extent to which the surfaces affect the heat transport process depends not only on the membrane thickness, but also on the thermal properties of the membrane system. Due to the high value of the thermal conductivity of the membrane, the heat resistance in the membrane is small. Then, the heat transfer resistance at the feed-membrane surfaces dominates the overall thermal resistance of the system, being the rate-determining step. 


\begin{tabular}{llll}
\hline $\begin{array}{l}\text { Membrane } \\
\text { thickness }(\mu \mathrm{m})\end{array}$ & $\begin{array}{l}\Delta_{l, m} T / \Delta_{\text {total }} T \\
(\%)\end{array}$ & $\begin{array}{l}\Delta T / \Delta_{\text {total }} T \\
(\%)\end{array}$ & $\begin{array}{l}\Delta_{m, r} T / \Delta_{\text {total }} T \\
(\%)\end{array}$ \\
\hline 150 & 63 & 33 & 4 \\
100 & 71 & 24 & 5 \\
50 & 81 & 14 & 5 \\
25 & 87 & 8 & 5 \\
10 & 91 & 3 & 6 \\
\hline
\end{tabular}

Table 4. Relative contributions to temperature difference over the whole system for membranes with different thicknesses. The temperature, $T^{l, m}$, of the feed mixture is $308 \mathrm{~K}$. The relative temperature difference over the $l$-side membrane surface is $\Delta_{l, m} T / \Delta_{\text {total }} T$. The relative temperature difference over membrane is $\Delta T / \Delta_{\text {total }} T$. The relative temperature difference over the $r$-side membrane surface is $\Delta_{m, r} T / \Delta_{\text {total }} T$.

\begin{tabular}{llll}
\hline $\begin{array}{l}\text { Membrane } \\
\text { thickness }(\mu \mathrm{m})\end{array}$ & $\begin{array}{l}\Delta_{l, m} a_{A} / \Delta_{\text {total }} a_{A} \\
(\%)\end{array}$ & $\begin{array}{l}\Delta a_{A} / \Delta_{\text {total }} a_{A} \\
(\%)\end{array}$ & $\begin{array}{l}\Delta_{m, r} a_{A} / \Delta_{\text {total }} a_{A} \\
(\%)\end{array}$ \\
\hline 150 & 4 & 94 & 2 \\
100 & 6 & 91 & 3 \\
50 & 10 & 81 & 9 \\
25 & 17 & 67 & 16 \\
10 & 28 & 45 & 27 \\
\hline
\end{tabular}

Table 5. Relative contributions to water activity difference over the whole system for membranes with different thicknesses. The water activity, $a_{A}^{l, m}$, of the feed mixture is 0.2213 . The relative activity difference over the $l$-side membrane surface is $\Delta_{l, m} a_{A} / \Delta_{\text {total }} a_{A}$. The relative activity difference over membrane is $\Delta a_{A} / \Delta_{\text {total }} a_{A}$. The relative activity difference over the $r$-side membrane surface is $\Delta_{m, r} a_{A} / \Delta_{\text {total }} a_{A}$.

Table 5 gives the relative contributions to water activity difference over the membrane system for membranes with different thicknesses. It is found that the main activity difference drop over the system is generated over the membrane for the thickest membranes. However, it is observed that the role of the surface becomes significant, and even dominant, as the membrane thickness decreases. For the membrane with a thickness of $150 \mu \mathrm{m}$, it is found that $94 \%$ of the water activity drop over the system is gen- 
erated over the membrane, while this difference is $6 \%$ over the surfaces. In contrast, for the membrane with a thickness of $10 \mu \mathrm{m}$, it is found that only $45 \%$ of the water activity drop over the system is generated over the membrane, while this difference is $55 \%$ over the surfaces. The extent to which the surfaces affect the overall mass transport resistance depends not only on the membrane thickness, but also on the permeability of the membrane. For highly permeable membranes, the mass transfer resistance at the feed and permeate boundary layers contributes significantly to the overall transport resistance, being the rate-determining step depending on the existing hydrodynamic conditions. For less permeable membranes, the overall mass transfer resistance is dominated by the membrane resistance, which becomes significant with increasing the membrane thickness. Moreover, there is a limiting membrane thickness, below which the contribution of the boundary layers resistance to the overall resistance can be larger than the membrane resistance. In the present study, calculations have shown that the membrane resistance contribution to the overall transport resistance is larger than that of the surfaces resistance. However, it was found that when thinner membranes were used, the surface resistance was larger than the membrane resistance. The turning point can be localized between 25 and $10 \mu \mathrm{m}$ of membrane thickness.

Results have shown that both temperature and activity differences over the membrane increase with the membrane thickness. These results are in agreement with the fact that, as the membrane thickness is increased, the contribution of the membrane resistance becomes greater. The permeation rate is inversely proportional to membrane thickness because permeation resistance enhances as membrane thickness increases. With an increase in the thickness membrane, the component molecules have to cross more paths inside the membrane and mass transfer becomes dominated by the slower mass transfer, so permeation flux decreases. Similarly, the heat flux in the membrane is dominated by the conductive contribution over the thermo-diffusive contribution. Then, a thicker membrane gives more heat transport resistance, and thereby decreases the heat flux and, as a consequence, increases the temperature difference over the membrane.

\section{Effect of temperature on the permeate side}

Recently, it has been shown that the process intensification approach can be used to improve the performance of a membrane process [33]. It is possible to heat selectively by microwaves one part of a membrane system to en- 
hance the evaporation of the desired component of a mixture. In the present study, the effect of adding heat at a local position is studied by replacing the temperature as a variable by thermostating the permeate side of the membrane. This effect is modeled by solving the coupled mass and heat transport equations (Eqs. (7), (15), and (16)) subject to the additional boundary condition $T=T^{m, r}$ at $x=\delta$, where $T^{m, r}$ is a predetermined value. In the calculations, the membrane feed temperature, $T^{m, l}$, is set to $305 \mathrm{~K}$, whereas the membrane permeate temperature, $T^{m, r}$, is set to the following values: $305 \mathrm{~K}, 315 \mathrm{~K}, 325 \mathrm{~K}$, and $335 \mathrm{~K}$. After calculations, it is found that the activity of water, $a_{A}^{r, m}\left(T^{m, r}\right)$, is decreased when the membrane permeate temperature increases, whereas the activity of ethanol, $a_{B}^{r, m}\left(T^{m, r}\right)$, increases with the membrane permeate temperature. However, the effect of adding heat at the permeate side is negligible; because the effect of the variation of the membrane permeate temperature on the component activities in the membrane is less than $1 \%$. This behavior may be due to the small values of the measurable heat of transfer, $q_{i}^{* m}$, of water and ethanol in the membrane.

\section{NET model validation}

Experimental data on temperature variation and heat flux across the membrane for the ethanol-water system analyzed in the present study are not reported in the literature. Hence, in order to validate our NET model, its predictions are compared to the experimental data provided by Rautenbach and Albrecht for isopropanol dehydration by pervaporation [24,25]. On the basis of their reported data, the heat flux as a function of the temperature difference over the membrane system can be estimated for the propanolwater system, and the results are shown in Figure 4. The NET model predictions were obtained according to the next procedure: the systems of equations representing the NET model - Eqs. (1), (2), (3), (7), (15), (16), (17), (18), and (19) - were solved using transport parameters and boundary conditions equal to those of the experimental propanol-water system. The membrane is in contact with binary mixture with a water content of 10 wt. $\%$, at a temperature, $T^{l, m}$, of $308 \mathrm{~K}$. The transport equations were solved using different values of the measurable heat flux, $J_{q}^{\prime l}$ (in $\mathrm{W} \cdot \mathrm{m}^{-2}$ : $680,1360,2040,2720$, and 3400). The NET data obtained in this way are also plotted in Figure 4. It is found that the NET approach is deemed good because the temperature data provided by the model agree very well to the experimental data. 


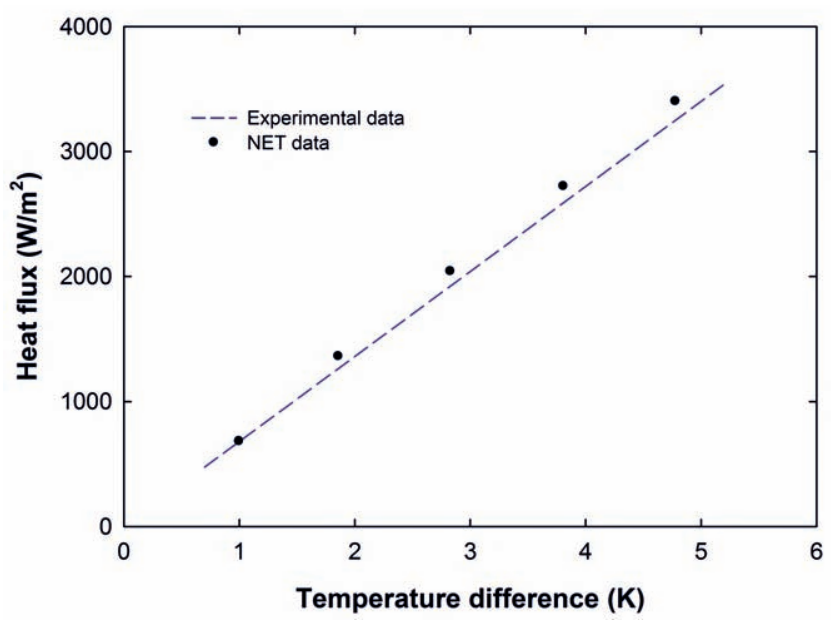

Figure 4. Experimental heat flux across the membrane for isopropanol dehydration as a function of the temperature difference (from the bulk feed-side to the permeate side close to the membrane surface). The measurable heat flux in the feed side $\left(J_{q}^{\prime l}: 680,1360,2040,2720\right.$, and $\left.3400 \mathrm{~W} \cdot \mathrm{m}^{-2}\right)$ as a function the temperature difference over the entire system $\left(T^{l, m}-T^{r, m}\right)$ predicted by NET model.

\section{Conclusions}

Coupled heat and mass transport equations are derived based on non-equilibrium thermodynamics to model the pervaporation of a water-ethanol mixture in a membrane. The calculated water and ethanol activity and temperature profiles decrease linearly across the membrane, and show discontinuities at both the feed-membrane and the membrane-permeate interfaces. The temperature difference over the system is dominated by the interfaces. In contrast, the component activity difference over the system is dominated by the membrane.

The influence of the membrane thickness on transport equations shows that the role of the feed-membrane and membrane-permeate surfaces becomes greater as the membrane thickness decreases. The calculated temperature data by the non-equilibrium thermodynamics model are comparable to experimental results available for water-alcohol systems. 


\section{Bibliography}

[1] Wijmans, J. G. and Baker, R. W., The solution-diffusion model: A review, J. Membr. Sci., 107 (1995), 1-21.

[2] Feng, X. and Huang, R. Y. M., Liquid separation by membrane pervaporation, Ind. Eng. Chem. Res., 16 (1997), 1048-1066.

[3] Okada, T. and Matsuura, T., A new transport model for pervaporation, J. Membr. Sci., 59 (1991), 133-150.

[4] Okada, T., Yoshikawa, M. and Matsuura, T., A study on the pervaporation of ethanol/water mixtures on the basis of pore flow model, J. Membr. Sci., 59 (1991), 151-168.

[5] Okada, T. and Matsuura, T., Predictability of transport equations for pervaporation on the basis of pore-flow mechanism, J. Membr. Sci., 70 (1992), $163-175$.

[6] Hwang, S. T., Nonequilibrium thermodynamics of membrane transport, AIChE J., 50 (2004), 862-870.

[7] Bedeaux, D. and Kjelstrup, S., Irreversible thermodynamics - A tool to describe phase transitions far from global equilibrium, Chem. Eng. Sci., 59 (2004), 109-118.

[8] Kjelstrup, S. and de Koeijer, G. M., Transport equations for distillation of ethanol and water from entropy production rate, Chem. Eng. Sci., 58 (2003), $1147-1161$.

[9] Kuhn, J., Stemmer, R., Kapteijn, F., Kjelstrup, S. and Gross, J., A nonequilibrium thermodynamics approach to model mass and heat transport for water pervaporation through a zeolite membrane, J. Membr. Sci., 330 (2009), 388-398.

[10] Inzoli, I., Simon, J. M., Kjelstrup, S. and Bedeaux, D., Thermal effects during adsorption of n-butane on a silicalite-1 membrane: A non-equilibrium molecular dynamics study, J. Colloid Interface Sci., 313 (2007), 563-573.

[11] Kjelstrup, S. and Bedeaux, D., Non-Equilibrium Thermodynamics of Heterogeneous Systems, Series on Advances in Statistical Mechanics, Volume 16, World Scientific Publishing, Singapore, 2008.

[12] Bolto, B., Hoang, M. and Xie, Z., A review of membrane selection for the dehydration of aqueous ethanol by pervaporation, Chem. Eng. Process., 50 (2011), 227-235. 
[13] Shao, P. and Huang, R. Y. M., Polymeric membrane pervaporation, J. Membr. Sci., 287 (2007), 162-179.

[14] Chapman, P. D., Oliveira, T., Livingston, A. G. and Li, K., Membranes for the dehydration of solvents by pervaporation, J. Membr. Sci., 318 (2008), 5-37.

[15] Xu, J., Kjelstrup, S., Bedeaux, D., Rosjorde, A. and Rekvig, L., Verification of Onsager's reciprocal relations for evaporation and condensation using non-equilibrium molecular dynamics, J. Colloid Interface Sci., 299 (2006), $452-463$.

[16] Burshe, M. C., Sawant, S. B., Joshi, J. B. and Pangarkar, V. G., Sorption and permeation of binary water-alcohols systems through PVA membranes crosslinked with multifunctional crosslinking agents, Sep. Purif. Technol., 12 (1997), 145-156.

[17] Varghese, J. G., Kittur, A. A., Rachipudi, P. S. and Kariduraganavar, M. Y., Synthesis, characterization and pervaporation performance of chitosang-polyaniline membranes for the dehydration of isopropanol, J. Membr. Sci., 364 (2010), 111-121.

[18] Magalad, V. T., Supale, A. R., Maradur, S. P., Gokavi, G. S. and Aminabhavi, T. M., Preyssler type heteropolyacid-incorporated highly waterselective sodium alginate-based inorganic-organic hybrid membranes for pervaporation dehydration of ethanol, Chem. Eng. J., 159 (2010), 75-83.

[19] Magalad, V. T., Gokavi, G. S., Raju, K. V. S. N. and Aminabhavi, T. M., Mixed matrix blend membranes of poly(vinyl alcohol)-poly(vinyl pyrrolidone) loaded with phosphomolybdic acid used in pervaporation dehydration of ethanol, J. Membr. Sci., 354 (2010), 150-161.

[20] Kjelstrup, S., Glavatsky, K. and Phoroah, J., Dynamic membrane boundary conditions for transport of heat and water, Proceeding of Fundamentals and Developments of Fuel Cells, Nancy, 2008.

[21] Platten, J. K., The Soret effect: A review of recent experimental results, J. Appl. Mech. Trans. ASME., 73 (2006), 5-15.

[22] Fang, G. and Ward, C. A., Temperature measured close the interface of an evaporating liquid, Phys. Rev. E., 59 (1999), 417-428.

[23] Simon, J. M., Kjelstrup, S., Bedeaux, D. and Hafskjold, B., Thermal flux through a surface of n-octane. A non-equilibrium molecular dynamics study, J. Phys. Chem. B., 108 (2004), 7186-7195. 
[24] Rautenbach, R. and Albrecht, R., On the behavior of asymmetric membranes in pervaporation, J. Membr. Sci., 19 (1984), 1-22.

[25] Rautenbach, R. and Albrecht, R., The separation potential of pervaporation. Part 2. Process design and economics, J. Membr. Sci., 25 (1985), 25-54.

[26] Villaluenga, J. P. G. and Cohen, Y., Numerical model of non-isothermal pervaporation in a rectangular channel, J. Membr. Sci., 260 (2005), 119-130.

[27] Vie, P. J. S. and Kjelstrup, S., Thermal conductivities from temperature profiles in the polymer electrolyte fuel cell, Electrochim. Acta, 49 (2004), 10691077.

[28] Burheim, O., Vie, P. J. S., Pharoah, J. G. and Kjelstrup, S., Ex situ measurements of through-plane thermal conductivities in a polymer electrolyte fuel cell, J. Power Sources, 195 (2010), 249-256.

[29] Villaluenga, J. P. G., Seoane, B., Barragán, V. M. and Ruiz-Bauzá, C., Thermo-osmosis of mixtures of water and methanol through a Nafion membrane, J. Membr. Sci., 274 (2006), 116-122.

[30] Jiraratananon, R., Chanachai, A. and Huang, R. Y. M., Pervaporation dehydration of ethanol-water mixtures with chitosan/hydroxyethylcellulose (CS/HEC) composite membranes. II. Analysis of mass transport, J. Membr. Sci., 199 (2002), 211-222.

[31] Schaetzel, P., Vauclair, C., Nguyen, Q. T. and Bouzerar, R., A simplified solution-diffusion theory in pervaporation: The total solvent volume fraction model, J. Membr. Sci., 244 (2004), 117-127.

[32] Yoshikawa, M., Masaki, K. and Ishikawa, M., Pervaporation separation of aqueous organic mixtures through agarose membranes, J. Membr. Sci., 205 (2002), 293-300.

[33] Drioli, E., Stankiewicz, A. I. and Macedonio, F., Membrane engineering in process intensification. An overview, J. Membr. Sci., 380 (2011), 1-8.

Received January 19, 2012; accepted June 12, 2012.

\section{Author information}

Juan P. G. Villaluenga, Department of Applied Physics, University Complutense of Madrid, Plaza de las Ciencias, 1, 28040 Madrid, Spain.

E-mail: juanpgv@fis.ucm.es

Signe Kjelstrup, Department of Chemistry, Norwegian University of Science and Technology, Realfagbygget, D3, NO-7491 Trondheim, Norway.

E-mail: signe.kjelstrup@nt.ntnu.no 
Copyright of Journal of Non-Equilibrium Thermodynamics is the property of De Gruyter and its content may not be copied or emailed to multiple sites or posted to a listserv without the copyright holder's express written permission. However, users may print, download, or email articles for individual use. 